\title{
Pancreatitis aguda recidivante con enteropatía por gluten asociada. Características clínico-analíticas y evolutivas en 34 pacientes
}

\author{
L. Rodrigo, N. Álvarez, S. Riestra, R. de Francisco, O. González Bernardo, L. García Isidro, \\ A. López Vázquez ${ }^{1}$ y C. López Larrea ${ }^{1}$
}

Servicios de Digestivo e ${ }^{1}$ Inmunología. Hospital Universitario Central de Asturias (HUCA). Oviedo, Asturias

\section{RESUMEN}

Objetivos: describir la frecuencia y características clínico-analíticas de la pancreatitis aguda (PA) recidivante con enteropatía por gluten (EG) asociada.

Pacientes y métodos: estudiamos de forma prospectiva los casos de pancreatitis agudas ingresados en nuestro Servicio durante el año 2006. Registramos un total de 185 pacientes. A las formas recurrentes que fueron 40 en total (22\%), les aplicamos un protocolo clínico-analítico consistente en la determinación de marcadores serológicos, genéticos y biopsias duodenales, para descartar una EG asociada.

Resultados: un total de 34 pacientes (18\%) cumplían criterios clínico-biológicos de EG asociada (grupo 1) y se compararon con el resto de las PA no-EG ( $\mathrm{n}=161$ ) (grupo 2). La edad media en la EG fue de $54 \pm 25$ años, ligeramente inferior al grupo 2, (61 \pm 14) (NS). Existía un ligero predominio de mujeres (50\%) en el grupo 1 , respecto al grupo $2(38,5 \%)$ (NS). Siete pacientes del grupo 1 (20\%) presentaron una PA grave, frente a 27 (17\%) en el grupo 2 (NS). La presencia de colelitiasis en el grupo 1, fue de 6 casos (18\%), significativamente inferior a la del grupo 2, de 72 casos $(45 \%)(p<0,05)$. Cuatro pacientes con EG desarrollaron seudoquistes (12\%) frente a $13(8 \%)$ en el grupo 2 (NS).

La transglutaminasa tisular (TGt) estaba elevada únicamente en 3 casos (9\%). Nueve pacientes (34\%) fueron DQ2 (+) y $4(12 \%)$ DQ8 (+), siendo el resto (54\%), negativos para ambos marcadores. Existía una duodenitis difusa desde el punto de vista endoscópico en 32 pacientes (95\%). Las biopsias duodenales, mostraron atrofia vellositaria (Marsh 3) en 2 casos (6\%); infiltración inflamatoria de la submucosa (Marsh 2) en 10 casos (29,4\%); aumento de los linfocitos intraepiteliales (Marsh 1) en 8 casos $(23,5 \%)$ y mucosa normal (Marsh 0) en 14 casos (41,2\%). La respuesta a la DSG al año, fue excelente en 30 pacientes (88\%).

Conclusiones: la PA recidivante con EG, constituye una asociación relativamente frecuente, indistinguible desde el punto de vista clínico y evolutivo del resto de PA, excepto por una menor presencia de colelitiasis $(p<0,05)$.

\begin{abstract}
Objectives: to describe the frequency and the clinical and laboratory characteristics of relapsing acute pancreatitis (AP) associated with gluten enteropathy (GE).

Patients and methods: we prospectively examined all acute pancreatitis cases admitted to our Department in 2006. We recorded a total of 185 patients. With recurring forms, 40 (22\%) in all, we used a clinical-lab protocol including serologic and genetic markers, and duodenal biopsy to rule out GE.

Results: a total of 34 patients (18\%) met clinical-biological criteria for GE (group 1), and were compared to the remaining nonGE AP cases ( $\mathrm{n}=161$ ) (group 2). Mean age in the GE group was $54 \pm 25$ years, slightly younger than group $2(61 \pm 14)$ (NS). There was a mild predominance of women (50\%) in group 1 versus group 2 (38.5\%) (NS). Seven patients in group 1 (20\%) had severe AP, as compared to $27(17 \%)$ in group 2 (NS). The presence of cholelithiasis in group 1 involved 6 cases (18\%), which was significantly lower than in group $2-72$ cases (45\%) (p < $0.05)$. Four patients with GE developed pseudocysts (12\%) versus $13(8 \%)$ in group 2 (NS).

Tissue transglutaminase (tTG) was elevated only in 3 patients (9\%). Nine patients (34\%) were DQ2 (+) and $4(12 \%)$ DQ8 (+); the rest (54\%) were all negative for both markers. From an endoscopic perspective there was diffuse duodenitis in 32 patients (95\%). Duodenal biopsies revealed villous atrophy (Marsh 3) in 2 patients (6\%); submucosal inflammatory infiltration (Marsh 2) in 10 (29.4\%); increased intraepithelial lymphocytes (Marsh 1) in 8 cases (23.5\%), and normal mucosa (Marsh 0) in 14 patients (41.2\%). Response to GFD after 1 year was excellent in 30 patients (88\%).

Conclusions: relapsing AP with GE represents a relatively common association that is indistinguishable from other APs from a clinical-evolutive standpoint, except for a lower presence of cholelithiasis $(p<0.05)$.
\end{abstract}


En estos pacientes es muy conveniente la realización de un protocolo diagnóstico específico para su identificación, ya que la DSG constituye la única medida eficaz, para prevenir la aparición de nuevos episodios de PA.

Palabras clave: Enfermedad celiaca. Pancreatitis aguda.
A specific diagnostic protocol is much needed in the identification of these patients since GFD is the only effective therapy to prevent new AP events from developing.

Key words: Celiac disease. Acute pancreatitis.

Rodrigo L, Álvarez N, Riestra S, de Francisco R, González Bernardo O, García Isidro L, López Vázquez A, López Larrea C. Pancreatitis aguda recidivante con enteropatía por gluten asociada. Características clínico-analíticas y evolutivas en 34 pacientes. Rev Esp Enferm Dig 2008; 100: 746-751.

\section{INTRODUCCIÓN}

La pancreatitis aguda (PA) es una enfermedad relativamente frecuente, que afecta aproximadamente a unos 300.000 pacientes al año, en Estados Unidos, con una mortalidad aproximada del 5\% (1). En España su incidencia media es muy similar y se estima aproximadamente en unos 35-40 casos/100.000 habitantes/año (2).

Se puede manifestar clínicamente de diversas maneras, siendo la más habitual el que se trate de un episodio único aislado, pero también con una cierta frecuencia se presenta en forma de ataques recurrentes, en la variedad denominada como "PA recidivante", o más raramente, como dolor abdominal crónico. Puede ser originada por causas muy diversas, entre las cuales las dos más frecuentes son las relacionadas con la presencia de colelitiasis y/o coledocolitiasis seguida en orden de frecuencia por el consumo excesivo de alcohol, representando entre ambas, alrededor del $75 \%$ de los casos.

Existen otras muchas posibles etiologías entre las que se incluyen los traumatismos abdominales, algunos fármacos, la hipertrigliceridemia, la isquemia mesentérica, la pancreatitis autoinmune, anomalías ductales pancreáticas congénitas como el páncreas divisum o adquiridas, así como por obstrucción mecánica al flujo pancreático debida a cáncer de páncreas o trastornos inflamatorios asociados. Todas ellas en su conjunto representan aproximadamente un $10 \%$ de los casos (3). Sin embargo, en alrededor de un $15 \%$ de los casos, la etiología de la PA permanece desconocida, a pesar de realizar diversos estudios de imagen y analíticos, para tratar de aclararlo.

Algunos casos de PA recidivante son debidos a la presencia de una obstrucción al drenaje del conducto pancreático secundarios a la presencia de una estenosis papilar o a una disfunción del esfínter de Oddi (DEO) (4). La insuficiencia pancreática y la pancreatitis recidivante se han puesto en relación con la presencia de una enteropatía por gluten (EG) asociada. Tradicionalmente se pensaba que ambas entidades podían estar relacionadas, como consecuencia de la existencia de una deficiencia nutricional presente en la intolerancia al gluten, ya que tanto la función exocrina pancreática, como la endocrina, suelen estar disminuidas, tanto en niños, como en adultos que presentan malnutrición. Actualmente se postula más bien, que los trastornos tanto pancreáticos como biliares, que aparecen asociados con la EG, pueden ser secundarios a la presencia de inflamación duodenal y de estenosis papilar secundaria asociada (5-10).

\section{PACIENTES Y MÉTODOS}

Se llevó a cabo un estudio prospectivo de los casos de PA ingresados en nuestro Servicio de Digestivo durante el año 2006, registrando un total de 185 pacientes.

De ellos separamos las PA recidivantes, definidas como haber tenido 2 o más episodios de agudización, representando un total de 40 pacientes.

El diagnóstico de PA se estableció en base a criterios clínico-biológicos admitidos, basados en la presencia de dolor abdominal persistente irradiado en cinturón hacia la espalda y acompañado de la elevación de enzimas pancreáticos en suero (amilasa y/o lipasa) con valores superiores al menos tres veces el límite superior de la normalidad.

Entre las pruebas de imagen para valorar morfológicamente el estado de la glándula pancreática, realizamos en primer lugar la ecografía abdominal, para comprobar la presencia o ausencia de colelitiasis, completada posteriormente en los casos necesarios, por la realización de un TAC dinámico, para la evaluación de la presencia y extensión de la necrosis pancreática y/o de las complicaciones locales asociadas.

En caso de sospecha de colangitis, coledocolitiasis, ictericia y /o obstrucción papilar, se procedió a la realización de una CPRE con esfinterotomía, en caso necesario.

Para la evaluación de los criterios de gravedad, se utilizó el sistema de clasificación Apache II, con inclusión de la obesidad como factor independiente de mal pronóstico, siendo su valoración mayor de seis, indicativa de riesgo de gravedad y por tanto de monitorización intensiva del paciente y aplicación de las medidas terapéuticas específicas oportunas. 
Todos los pacientes recibieron tratamiento estándar, basado en las medidas habituales analgésicas, reposición hidroelectrolítica adecuada, soporte nutricional precoz y tratamiento oportuno de las complicaciones

Para el estudio de la intolerancia al gluten asociada, se determinaron los anticuerpos anti-transglutaminasa (TGt) séricos por técnica de ELISA comercial (Orgentec, Diagnostica, Mainz, Alemania), considerando valores positi$\operatorname{vos}(>1 \mathrm{U} / \mathrm{ml})(11)$.

Asimismo se realizaron las determinaciones genéticas de los marcadores de susceptibilidad para la EG, del sistema HLA-II (DQ2 y DQ8) por técnicas de PCR-SSP habituales (12). La toma de biopsias duodenales múltiples, se llevó a cabo mediante duodenoscopia realizada con endoscopios Fujinon EC-250WR, tomadas a nivel de la segunda porción duodenal y se clasificaron de acuerdo con los estadios de Marsh (13).

Fueron diagnosticados de enteropatía sensible al gluten (EG) todos aquellos pacientes que presentaban rasgos clínicos y/o analíticos compatibles, junto con la presencia de marcadores genéticos de susceptibilidad y/o marcadores serológicos positivos (TGt elevada) y/o lesiones histológicas duodenales compatibles y/o respuesta a la dieta sin gluten de forma mantenida, más de seis meses. Dentro de ella se incluyen las denominadas formas latente y potencial de la enfermedad celiaca (EC).

Se instauró una dieta sin gluten (DSG) a los pacientes así diagnosticados de EG y se siguió su respuesta clínicoanalítica cada seis meses, en controles ambulatorios, siendo valorada su evolución al cabo de un año de seguimiento.

\section{Análisis estadístico}

Las variables continuas se representan como medias y desviación estándar. Para las variables categóricas se utilizaron los porcentajes. Para el análisis de las primeras se utilizó la t de Student y para las segundas, el de la Chi cuadrado, con la corrección de Yates, o la prueba exacta de Fisher, cuando se estimó necesario. Se consideraron diferencias significativas entre grupos, cuando el valor de $\mathrm{p}$ fue menor de 0,05 .

\section{RESULTADOS}

Del total de 185 casos de pancreatitis agudas, ingresadas en el Servicio de Digestivo de nuestro hospital, durante el año 2006, 40 casos $(21,6 \%)$ cumplían los criterios de formas recidivantes. A todas ellas se les aplicó un protocolo clínico-biológico para descartar una EG asociada y encontramos un total de 34 pacientes, lo que representa un $18,3 \%$ del total de pacientes con PA y el $85 \%$ (34/40) de las formas recidivantes.

Se compararon los pacientes que presentaban una EG asociada $(\mathrm{n}=34)$ (grupo 1$)$, con los restantes pacientes con PA sin EG $(n=161)$ (grupo 2).
La edad media los pacientes del grupo 1, fue de $54 \pm$ 25 años (24-82) y estaban equitativamente repartidos por sexos 17 casos (50\%). Un 44\% presentaban edades superiores a la $6^{\mathrm{a}}$ década de la vida, al momento del diagnóstico. La edad media de los pacientes del grupo 2, fue ligeramente superior y había una menor proporción de mujeres (NS).

En cuanto a las formas clínicas es de resaltar que 7 pacientes $(20 \%)$ presentaron formas graves de evolución, por lo que precisaron ingreso en la Unidad de Cuidados Intensivos en el grupo 1 y 27 (17\%) en el grupo 2, sin diferencias significativas.

Seis pacientes (18\%) presentaron colelitiasis en el grupo 1 , frente a $72(45 \%)$ del grupo 2 , siendo esta la única diferencia significativa encontrada entre ambos $(\mathrm{p}<$ $0,05)$.

A cuatro pacientes del grupo 1, se les practicó colecistectomía por colelitiasis asociada, resaltando que todos ellos recidivaron de su PA, a pesar de la extirpación de la vesícula sin presentar coledocolitiasis acompañante.

La CPRE se realizó en 4 pacientes (12\%) del grupo 1 frente a $24(15 \%)$ del grupo 2 (NS).

En 4 pacientes (12\%) se realizó esfinterotomía endoscópica (EE) por CPRE y en 2 de ellos $(6 \%)$ se produjo una perforación duodenal iatrógena, que se resolvió en ambos casos, con tratamiento conservador.

Es de reseñar igualmente la presencia de complicaciones locales ya que 4 pacientes $(12 \%)$ presentaron seudoquistes en el grupo 1, frente a $13(8 \%)$ en el grupo 2 (NS).

Las características generales, clínicas y la incidencia de complicaciones entre ambos grupos se describen en la tabla I.

Tabla I. Características generales, clínicas y complicaciones de las PA con y sin EG asociada

\begin{tabular}{lccc}
\hline Características & $\begin{array}{c}\text { PA con EG asociada } \\
(n=34)\end{array}$ & $\begin{array}{c}\text { PA sin EG } \\
(n=161)\end{array}$ & Valor de $p$ \\
\hline Edad media, años & $54 \pm 25$ & $61 \pm 18$ & NS \\
Mujeres, $n(\%)$ & $17(50)$ & $62(38,5)$ & NS \\
Formas graves & $7(20)$ & $27(17)$ & NS \\
Colelitiasis, $n(\%)$ & $6(18)$ & $72(45)$ & $<0,05$ \\
CPRE, $n(\%)$ & $4(12)$ & $24(15)$ & NS \\
Colecistectomías $n(\%)$ & $6(17)$ & $30(19)$ & NS \\
Seudoquistes, $n(\%)$ & $4(12)$ & $13(8)$ & NS \\
\hline
\end{tabular}

PA: pancreatitis agudas; EG: enteropatía por gluten; CPRE: colangiopancreatografía retrógrada endoscópica; NS: no significativo.

Una paciente del grupo $1(3 \%)$, fue intervenida realizándole una resección de la cabeza pancreática por técnica de Whipple por dolor persistente. Otro paciente (3\%) fue intervenido para limpieza y drenaje de absceso pancreático, que no respondió a tratamiento antibiótico intenso. No se registró ningún caso de mortalidad en la presente serie. En la endoscopia digestiva alta realizada a los 34 pacientes incluidos en el estudio, se informó de la presencia de signos macroscópicos de duodenitis difusa en 
la mayor parte de los pacientes, concretamente en 32 casos $(95 \%)$.

Los valores séricos de la transglutaminasa tisular (TGt) estaban elevados claramente en 3 casos (9\%); los restantes 31 casos $(91 \%)$, presentaban valores mínimamente elevados, o situados dentro del rango de la normalidad.

En cuanto a los marcadores genéticos de susceptibilidad al gluten, 9 pacientes (34\%) eran DQ2 positivos; 4 casos $(12 \%)$ eran DQ8 positivos y los restantes 21 pacientes (44\%) eran considerados DQ2 y DQ8 negativos, presentando algún alelo positivo de uno o ambos heterodímeros (Tabla II).

\begin{tabular}{lc}
\multicolumn{2}{c}{ Tabla II. Marcadores serológicos y genéticos en pacientes } \\
con PA y EG asociada $(\mathbf{n}=\mathbf{3 4 )}$ \\
\hline Transglutaminasa positiva & $3(9 \%)$ \\
HLA-DQ2 (+) & $9(34 \%)$ \\
HLA-DQ8 (+) & $4(12 \%)$ \\
\hline
\end{tabular}

PA: pancreatitis aguda; EG: enteropatía por gluten.

Respecto a los hallazgos histológicos de la biopsia duodenal predominaban los hallazgos inflamatorios de la mucosa y submucosa intestinal o cambios mínimos, (estadios 0-2 de Marsh), encontrando una clara atrofia vellositaria presente, únicamente en 2 pacientes (6\%) (Tabla III).

La respuesta a la dieta sin gluten (DSG) fue excelente al año de seguimiento, permaneciendo asintomáticos y sin recidiva de su PA, un total de 31 pacientes $(88 \%)$.

\begin{tabular}{lc}
$\begin{array}{c}\text { Tabla III. Clasificación de las biopsias duodenales (Marsh) en } \\
\text { pacientes con PA y EG asociada }(\mathbf{n}=\mathbf{3 4 )}\end{array}$ \\
\hline Estadio 0 & $14(41 \%)$ \\
Estadio 1 & $8(23 \%)$ \\
Estadio 2 & $10(30 \%)$ \\
Estadio 3 & $2(6 \%)$
\end{tabular}

PA: pancreatitis aguda; EG: enteropatía por gluten.

\section{DISCUSIÓN}

La asociación entre pancreatitis aguda con la EC, o intolerancia al gluten, es bien conocida, habiéndose descrito casos y series aisladas desde hace varios años $(14,15)$. De la misma manera se han descrito casos de pancreatitis crónica calcificada asociados con EC y anomalías congénitas como el páncreas divisum (16) o con desnutrición como en la denominada "pancreatitis tropical", que se presenta predominantemente en la India (17).
Estudios epidemiológicos recientes indican que esta asociación es mucho mayor de lo que inicialmente se pensaba. Así en un estudio publicado en 2007, llevado a cabo en Suecia en población general en un Registro Nacional seguido durante 40 años (1064-2003), se identificaron un total de 14.239 individuos que presentaban una enfermedad celiaca, comparado con 69.381 individuos controles de referencia de la misma edad, sexo y lugar de residencia, seguidos durante más de un año, sin diagnóstico previo de pancreatitis o un año posterior a la entrada en el estudio. En el periodo de seguimiento se pudo comprobar que los pacientes celiacos tuvieron un riesgo relativo de 3,3 (IC-95\%: 2,6-4,4) ( $<<0,001)$ de desarrollar pancreatitis aguda y mayor aún de presentar pancreatitis crónica, con una OR de 19,8 (IC-95\%: 9,2-42,8) (p < $0,0001)$. El riesgo aumentado de padecer pancreatitis se encontró únicamente en pacientes diagnosticados de EC, en la edad adulta. Por lo tanto este estudio demuestra de manera concluyente que los pacientes celiacos tienen un mayor riesgo de presentar pancreatitis, tanto aguda como crónica, a lo largo de su vida, que la población general, de forma significativa (18).

Los mecanismos postulados de la relación entre la EC y la patología inflamatoria pancreático-biliar incluyen la existencia de un vaciamiento lento de la vesícula debido a una disminución de liberación de la colecistoquinina e incluso una hipotética malnutrición. Pero hoy en día se postula más bien, que la causa principal está relacionada con la presencia de una inflamación duodenal difusa, que condiciona secundariamente la existencia de una estenosis papilar.

Así se comprobó en un estudio realizado por Patel y cols. (19) sobre 169 pacientes para estudio de una posible disfunción del esfínter de Oddi, a los que se les realizó una manometría pancreático-biliar. A todos ellos les determinaron también los anticuerpos antigliadina y antiendomisio, e igualmente les tomaron biopsias duodenales y papilares en aquellos pacientes con serología positiva para EC.

Encontraron 12 pacientes con EC $(7,1 \%)$, cuya edad media fue de 61 años, muy superior a la de los pacientes que no tenían EC que era de 37 años. Todos los pacientes celiacos, presentaban dolor abdominal recurrente y/o pancreatitis idiopática. Dos de ellos presentaban ligera elevación de las pruebas de función hepática y 10 tenían amilasa y/o lipasa elevadas. Sólo 3 de los 12 pacientes habían sido diagnosticados previamente de enfermedad celiaca. Todos los pacientes tenían evidencia manométrica de estenosis y existía una inflamación periampular, confirmada histológicamente. Los pacientes fueron tratados con DSG presentando una remisión clínica mantenida (19).

La enfermedad celiaca se caracteriza por tratarse de un proceso sistémico de naturaleza autoinmune, el único de etiología conocida, que afecta de forma principal aunque no exclusiva al tubo digestivo, preferentemente a la porción más proximal del intestino delgado, principalmente al duodeno. Dada esta base se ha pensado por algunos 
autores, que también se podría relacionar con la pancreatitis autoinmune que cursa con afectación bilio-pancreática y se caracteriza por aumento de los niveles séricos de IgG-4 y con positividad de los anticuerpos frente a la enzima anhidrasa carbónica (20).

Aunque se trata de un proceso con base genética bien conocida, puede aparecer a cualquier edad de la vida y hasta un $20 \%$ de los casos se diagnostican en pacientes mayores de 60 años, como ha ocurrido en la presente serie, en la que hasta un $44 \%$ de nuestros pacientes estaban

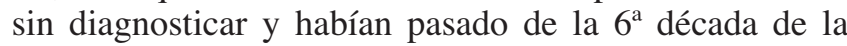
vida.

Hasta hace unos años, se consideraba que la EC era un proceso raro, pero actualmente es bien conocido que se trata de una enfermedad muy frecuente, con una distribución mundial bastante homogénea y con una prevalencia media del $1 \%$ en población general y que además está claramente subestimada e infradiagnosticada (21-23).

Las formas clínicas de presentación de la EC son muy variadas y en general menos características que en el niño (formas clásicas) por lo que se denominan "formas atípicas". La diarrea aparece en menos del $50 \%$ de los casos y muchos pacientes cursan con estreñimiento acentuado. La pérdida de peso está ausente o es poco llamativa y hasta un 30\% de los pacientes presentan sobrepeso en el momento del diagnóstico (24).

Se ha descrito gráficamente la EC como un "iceberg", en el que la parte visible y clínicamente manifiesta está constituida por las formas clásicas, que se presentan de forma predominante en la infancia, mientras que las formas atípicas del adulto, constituyen su parte sumergida u oculta, entre las que se incluyen las denominadas, formas silentes, latentes, potenciales y la enteropatía inducida por gluten (EG).

Otras manifestaciones clínicas frecuentemente asociadas con la EC son la presencia de anemia ferropénica, osteoporosis, alteraciones tiroideas, pruebas hepáticas alteradas, cefaleas, astenia y alteraciones cutáneas del tipo de la dermatitis herpetiforme que se presenta en un $25 \%$ de los celiacos, presentando lesiones cutáneas muy características por su típico aspecto vesículo-costroso, su presentación simétrica en zonas de roce y por el intenso prurito asociado, ayudando mucho su identificación para llegar al diagnóstico, ya que su presencia implica la existencia de una EC asociada, generalmente con sintomatología digestiva escasa o intermitente por la existencia de lesiones duodenales no atróficas, o de distribución parcheada, en el $100 \%$ de los casos (25).

En cuanto a los marcadores serológicos actualmente disponibles para orientar el diagnóstico de EC, el más utilizado es la transglutaminasa tisular (TGt), ya que se realiza mediante técnicas de Elisa comercial y muestra una alta sensibilidad y especificidad (cercanas al 90\%) en presencia de atrofia de vellosidades. Sin embargo, en ausencia de esta la sensibilidad baja mucho y sus resultados son negativos, como ocurrió en el presente estudio en que solamente fue positiva en 3 casos (9\%) ya que sólo 2 pacientes presentaban atrofia vellositaria de grado leve a moderado. Por ello es una prueba que tiene muy baja sensibilidad diagnóstica en los adultos, ya que guarda un paralelismo con la presencia de atrofia de las vellosidades intestinales y esta predomina claramente en las formas clásicas del niño y está generalmente ausente en los adultos, lo que justifica que la TGt sea habitualmente negativa. Su sensibilidad diagnóstica en el adulto, baja mucho con relación al niño, siendo del orden del $15-30 \%$ de los casos (26).

El espectro de los cambios histológicos que se presentan a nivel de la mucosa duodenal es muy amplio y no se limita, como tradicionalmente se pensaba, en encontrar la presencia de una atrofia de vellosidades intestinales de forma sistemática, sino que por el contrario, en los adultos este hallazgo es muy infrecuente y predominan los cambios mínimos que oscilan desde una mucosa intestinal morfológicamente normal (tipo 0 de Marsh), pasando por un incremento del número de linfocitos intraepiteliales generalmente superior al 30\% (tipo 1), a la presencia de una hiperplasia de criptas acompañada de un infiltrado inflamatorio difuso a nivel de la submucosa, como hallazgos más frecuentes en la EG del adulto, observando en la gran mayoría de los casos una excelente respuesta clínica a la administración continuada de una DSG, lo que confirma que se trata de auténticos pacientes celiacos (27-29).

Por todo ello, no puede extrañarnos el que, de momento, no dispongamos de un consenso general respecto a los criterios diagnósticos de la EC, principalmente en los referentes a la utilidad de los marcadores serológicos y de los parámetros histológicos utilizados, entre diversas guías clínicas elaboradas por diversos comités de expertos europeos e internacionales (30-32).

Existen grupos de riesgo para presentar una EC, que incluyen individuos que presentan enfermedades autoinmunes asociadas, tales como la diabetes tipo 1, las enfermedades tiroideas así como la alteración persistente de las pruebas de función hepática (33).

Entre los familiares de primer grado de pacientes con EC, existe una prevalencia aumentada de padecerla, que se estima en torno a un $10-15 \%$ y que incluye también a familiares de secundo grado, lo que habla claramente a favor de la predisposición genética y de la importante agrupación familiar para el padecimiento de esta enfermedad $(34,35)$.

A modo de resumen queremos resaltar la conveniencia de realizar un despistaje de EG en los casos de pacientes con PA recidivante, ya que se trata de una asociación relativamente frecuente y puede cursar con formas graves y complicadas, sin diferencias en relación con el resto de PA de otra etiología (biliar o alcohólica). Su diagnóstico no resulta sencillo, ya que los marcadores serológicos habitualmente empleados para el despistaje de la EG, son frecuentemente negativos y es muy conveniente, pese a ello, el realizar una endoscopia con toma de múltiples biopsias duodenales, preferentemente distales y periam- 
pulares. En casos dudosos está justificada la instauración de una dieta sin gluten, ya que produce en la mayoría de los casos una mejoría clínica significativa, con prevención de la aparición de nuevos episodios de recidiva de la pancreatitis aguda y/o crónica.

\section{BIBLIOGRAFÍA}

1. Cappell MS. Acute pancreatitis: etiology, clinical presentation, diagnosis, and therapy. Med Clin North Am 2008; 92: 889-923.

2. Mínguez M, García A, Boix V. Pancreatitis aguda. Estudio epidemiológico prospectivo en la provincia de Alicante. Grupo hospitalario para el estudio de las enfermedades digestivas. Rev Esp Enferm Dig 1995; 87: 869-73.

3. Aparisi L, Farré A, Gómez-Cambronero L, Martínez J, De las Heras $\mathrm{G}$, Corts J, et al. Antibodies to carbonic anhydrase and IgG4 levels in idiopathic chronic pancreatitis: relevance for diagnosis of autoimmune pancreatitis. Gut 2005; 54: 703-9.

4. Tarnaki PR, Hoffman B, Asbakken L, Knapple W, Coyle W, Pineau $\mathrm{R}$, et al. Sphincter of Oddi dysfunction is associated with chronic pancreatitis. Am J Gastroenterol 1997; 92: 1125-9.

5. Pitchumoni CS, Thomas E, Balthazar F, Sherling B. Chronic calcific pancreatitis in association with celiac disease. Am J Gastroenterol 1977; 68: 358-61.

6. Bustos Fernández L, De Paula A, Prizont R, Tiscornia OM, Braguinsky J, Carreras A, et al. Exocrine pancreatic insufficiency secondary to gluten enteropathy. Am J Gastroenterol 1970; 53: 564-9.

7. Benson GD, Kowlessar OD, Sleisenger MH. Adult celiac disease with emphasis upon response to a gluten free diet. Medicine 1963; 43: $1-40$.

8. Pink LJ, Ceamer B. Response to a gluten free diet of patients with celiac syndrome. Lancet 1967; 1: 300-4.

9. Fitzgerald O, Fitzgerald P, Fennelly, Mc Mullin JP, Boland SJ. A clinical study of chronic pancreatitis. Gut 1963; 4: 193-216.

10. Dimagno EP, Go VLW, Summerskill WHJ. Impaired cholecystokinin-pancreozymin secretion, intraluminal dilution and maldigestion of fat in sprue. Gastroenterology 1972; 63: 25-32.

11. Fernández E, Riestra S, Rodrigo L, Blanco C, López-Vázquez A, Fuentes D, et al. Comparison of six human anti-transglutaminase ELISA-tests in the diagnosis of celiac disease in the Sharawui population. World J Gastroenterol 2005; 11: 3762-6.

12. Olerup O, Aldener A, Fogdell A. HLA-DQB1 and DQA1 typing by PCR amplification with sequence specific primers (PCR-SSP) in two hours. Tissue Antigens 1993; 11: 119-34.

13. Marsh MN. Gluten, major histocompatibility complex, and the small intestine. A molecular and immunobiological approach to the spectrum of gluten sensitivity. Gastroenterology 1992; 102: 330-54.

14. Thomson A. Celiac disease as a cause of pancreatitis. Gastroenterology 2005; 129: 1137.

15. Hillman L. Coeliac disease and pancreatitis. Intern Med J 2005; 35 : 374-5.

16. Arya S, Rana SS, Sinha SK, Nagi B, Bhasin DK. Celiac disease and chronic calcific pancreatitis with pancreas divisum. Gastrointest Endosc 2006; 63: 1080-1.
17. Sood A, Midha V, Sood N, Bansal M, Kaur M, Goyal A, et al. Coexistence of chronic calcific pancreatitis and celiac disease Indian J Gastroenterol 2007; 26: 41-2.

18. Ludvigsson JF, Montgomery SM, Ekbom A. Risk of pancreatitis in 14,000 individuals with celiac disease. Clin Gastroenterol Hepatol 2007; 5: 1347-53.

19. Patel RS, Johlin FC, Murray JA. Celiac disease and recurrent pancreatitis. Gastrointest Endosc 1999; 50: 823-7.

20. Leeds JS, Sanders DS. Risk of pancreatitis in patients with celiac disease: is autoimmune pancreatitis a biologically plausible mechanism? Clin Gastroenterol Hepatol 2008; 6: 951.

21. Rodrigo L, Garrote JA, Vivas S. Enfermedad celiaca. Med Clin (Barc) 2008; 131: 264-70.

22. García Novo MD, Garfia C, Acuña Quirós MD, Asensio J, Zancada G, Barrio Gutierrez S, et al. Prevalencia de la enfermedad celíaca en donantes de sangre aparentemente sanos en la Comunidad Autónoma de Madrid. Rev Esp Enferm Dig 2007; 99: 337-42.

23. Riestra S, Fernández E, Rodrigo L, García S, Ocio G. Prevalence of celiac disease in the general population in northern Spain. Strategies of serologic screening. Scand J Gastroenterol 2000; 35: 398-402.

24. Lo W, Sano K, Lebwohl B, Diamond B, Green PH. Changing presentations of celiac disease. Dig Dis Sci 2003; 48: 395-8.

25. Green PH. The many faces of celiac disease: clinical presentation of celiac disease in the adult population. Gastroenterology 2005; 128 (Supl. 1): 74-8.

26. Abrams JA, Brar P, Diamond B, Rotterdam H, Green PH. Utility in clinical practice of immunoglobulin-A anti-tissue transglutaminase antibody for the diagnosis of celiac disease. Clin Gastroenterol Hepatol 2006; 4: 726-30.

27. Tursi A, Brandimarte G. The symptomatic and histologic response to a gluten-free diet in patients with borderline entheropathy. J Clin Gastroenterol 2003; 36: 13-7.

28. Kakar S, Nehra V, Murray JA, Dayharsh GA, Burgart LJ. Significance of intraepithelial lymphocytosis in small bowel biopsy samples with normal mucosa architecture. Am J Gastroenterol 2003; 98: 2027-33.

29. Rostami K, Kerchhaert J, Blomberg BME, Meijer JWR, Mulder CJJ. Sensitivity of antiendomysium and antigliadin antibodies in untreated celiacs: disappointing in clinical practice. Am J Gastroenterol 1999; 94: 888-94.

30. Walker-Smith JA, Guandalini S, Scmitz J, Shmerling DH, Visakorpi JK. Revised criteria for diagnosis of celiac disease. Report of the Working Group of the European Society of Paediatric Gastroenterology and Nutrition. Arch Dis Child 1990; 65: 909-11.

31. The UECW Working Group. When is a celiac a celiac? Report of a working group of the United European Gastroenterology Week, in Amsterdam, 2001. Eur J Gastroenterol Hepatol 2001; 13: 1-6.

32. Ciclitira PJ, Ling AL, Fraser JS. AGA technical review on coeliac sprue. Gastroenterology 2001; 120: 1526-40.

33. Collin P, Kaukinen K, Valimaki M, Salmi J. Endocrinological disorders and celiac disease. Endocr Rev 2002; 23: 464-83.

34. Rodrigo L, Riestra S, Fuentes D, González S, López-Vázquez A, López-Larrea C. Diversas formas clínicas de presentación de la enfermedad celíaca dentro de la misma familia. Rev Esp Enferm Dig 2004; 96: 612-9.

35. Rodrigo L, Fuentes D, Riestra S, Niño P, Álvarez N, López-Vázquez A, et al. Prevalencia aumentada de enfermedad celíaca en familiares de primer y segundo grado: descripción de una familia con 19 miembros estudiados. Rev Esp Enferm Dig 2007; 99: 149-55. 